\title{
Investigating the Use of Visualisations of Biomechanics in Physical Rehabilitation
}

\author{
David Loudon $^{1}$, Bruce Carse ${ }^{2}$, and Alastair S. Macdonald ${ }^{1}$ \\ ${ }^{1}$ The Glasgow School of Art, Glasgow, UK \\ \{d. loudon, a.macdonald\}@gsa.ac.uk \\ ${ }^{2}$ University of Strathclyde, Glasgow, UK \\ bruce.carseastrath.ac.uk
}

\begin{abstract}
Biomechanical analysis can be used to scientifically assess the causes of movement problems, measure progress and validate outcomes. However, the complexity of the data produced and the training required to use the available biomechanical analysis tools prevents the widespread understanding of this form of analysis beyond those with a background in biomechanics. This paper reports on multidisciplinary research, funded by the MRC's LLHW programme, into the generation of three-dimensional, dynamic visualisations of biomechanical data and investigation of their use during functional rehabilitation trials, e.g., post-stroke, knee-joint replacement, and older adult exercise. The research will test the hypothesis that increased understanding of biomechanical concepts and measurements through this method of visualisation by both patients and clinicians will result in better patient outcomes.
\end{abstract}

Keywords: Visualisation, Biomechanics, Rehabilitation.

\section{Introduction}

Biomechanical analysis can be used to scientifically assess the causes of movement problems and to measure rehabilitation progress and outcomes. However, at present biomechanics is only explicitly used in a small number of specialist rehabilitation scenarios. This paper reports on multidisciplinary research investigating the use of visualisation software to enable the application of biomechanical data and analysis in a wider range of rehabilitation settings.

The paper begins with a discussion of the benefits which biomechanical analysis can provide for rehabilitation, and the factors which currently limit its widespread use. The approach taken in the research to overcoming these limitations is then introduced: firstly, the potential for visualisation techniques to communicate complex, dynamic biomechanical data in an accessible way to those without biomechanics training; secondly, the investigation of the application of the visualisation software to five different rehabilitation scenarios, which cover a range of different rehabilitation settings and complexity of patient condition. In the last section, the implications of the different rehabilitation settings are discussed: hospital, community and home. 


\section{Biomechanics in Rehabilitation: Current Limitations and Potential Benefits}

The aim of physical rehabilitation is to work the musculoskeletal system in the most effective way to restore function and quality of life following injury or illness e.g. to rebuild soft tissue which has been damaged or underused; or enable relearning of motor control of muscles following brain injury.

Rehabilitation professionals therefore need to (a) assess the difficulties the patient is having with movement, and (b) determine and discuss with the patient realistic rehabilitation goals, and a programme of rehabilitation tasks which could restore their function to meet those targets.

Human movement is complex and dynamic. If you ask several people to perform the same functional task, each will achieve it by moving their body in a different way - these differences are often subtle and therefore difficult to see by eye. Biomechanics can provide highly accurate data on human movement beyond the immediately observable. However, to date biomechanical data measurement and analysis have only been used in a small number of rehabilitation scenarios. The most common clinical application for biomechanics is in gait analysis. Due to current biomechanical analysis systems, these are specialist sessions which are expensive both in terms of the equipment required and the number of staff needed to collect and interpret the results.

The following sections describe the limitations of current biomechanical analysis systems and software, and the potential benefits which could be provided for patients if these limitations could be overcome.

\subsection{Limiting Factors to Widespread Use of Biomechanics in Rehabilitation}

The biomechanical analysis systems currently available are highly accurate, and provide dedicated software to analyse the kinematic and kinetic parameters of movement. While the tools offer flexibility for specialists, there are a number of limitations to the systems which make them unsuitable for general clinical use.

- The analysis software is tightly integrated with expensive laboratory equipment limiting its use to only a small number of hospital facilities. The majority of therapists in the UK rarely have access to motion analysis equipment [1].

- Most therapists do not have training in biomechanics to the level required to collect motion analysis data and then use the software to perform an analysis.

- Interpretation of the data collected by motion analysis, particularly with clinical gait data, is also considered to be problematic [2]. "Interpretation of biomechanical data is complex, time consuming and not readily understood by most therapists" [3].

- Biomechanical analysis tools are designed to be general purpose, and are not tied to any particular rehabilitation application. This is beneficial in terms of the flexibility of the tools. However as a result, they provide all the data measurements from a motion without any guidance on which parameters are important or how they relate to each other. 
- The representations of biomechanical analyses of movement in current tools are of limited use when trying to communicate to those without a background in biomechanics - with other health professionals or the patient themselves. This is generally presented in the form of a generic 'gait report' which is thought to contain too many pages of text, graphs and figures [4].

\subsection{Potential Benefits of Biomechanical Analysis for Rehabilitation}

There are a number of potential benefits to the use of biomechanical analysis in rehabilitation settings. Motion analysis systems and supporting software enable the collection of accurate, repeatable and objective data on the complex dynamic movements patients perform. This type of analysis has shown a demonstrable positive effect on clinical decision-making in the context of gait analysis [5,6]. The ability to provide this data in other rehabilitation settings could enable clinicians to make more informed decisions.

While biomechanical analysis in rehabilitation can be beneficial to clinicians, it may also prove advantageous to patients as it could assist them with understanding their rehabilitation tasks, goals and progress.

The benefits of involving patients in their own care have been demonstrated [7, 8]. Patient understanding of their treatment and effective communication with clinicians have both been identified as having a positive impact on their compliance [9] which leads to a better chance of improved treatment outcomes [10].

Understanding plays a particularly important role in stroke rehabilitation. In one study, stroke patients identified as highly motivated stated that information from clinicians helped them to understand their goals, identify progress and take an active role in their own rehabilitation. Lack of understanding on the other hand was thought to contribute to non-compliance. Furthermore, the ability to discuss, or even challenge, the decisions with clinicians was described as important [11].

\section{Visualisations of Biomechanics: Applying Biomechanics to Specific Rehabilitation Scenarios}

The field of Information Visualisation offers a potential route to improving the communication of the complex and interrelated biomechanical parameters describing human movement. Visualisation uses visual representations and interaction techniques to use the processing power of the human eye to enable people to see, explore, and understand large amounts of information at once [12]. Further, it goes beyond simple presentation of data, but rather enables understanding the meaning of the data, or even has the ability to create new meaning which was not visible before [13]. Visualisation can provide new insights about data, supporting discovery, decision making and explanation [14].

In previous work by the authors $[15,16]$ the ability of visualisation techniques to communicate biomechanical data on the demands of activities of daily living for older adults were investigated. The research aimed to improve on the traditional approaches of using static graphs, tables and figures by producing three-dimensional, dynamic visualisations of biomechanical data. The research found that through the use of 
visualisation techniques the biomechanical data, which would usually have been inaccessible, could be understood by both lay and professional audiences. Further, the visualisations were shown to enable new cross-disciplinary dialogues about the data between the professionals and lay members.

While this research proved the potential value of visualisation to communicate biomechanical data to a wider audience, the data did not directly benefit the individual that was measured, so could not be fed back to the patient to help them to correct their movements or suggest exercises to improve their functional capability.

The envisage project, funded by the Lifelong Health and Wellbeing (LLHW2) initiative, will investigate the potential of visualisation software to address the challenges of using biomechanical data in rehabilitation. The project is a multidisciplinary collaboration between the University of Strathclyde, The Glasgow School of Art and Glasgow Caledonian University.

Five discrete work packages have been selected to investigate the use of visualisations of biomechanical data in a range of rehabilitation settings, involving different treatment processes and complexity of condition.

- Falls prevention advice and visual feedback to those at risk of falling. (Home-based)

- Functional exercises for the rehabilitation of total knee replacement patients. (Home-based)

- Lower limb stroke rehabilitation for acute stroke patients. (Communitybased)

- Upper limb stroke rehabilitation for acute stroke patients. (Communitybased)

- Diagnosis and fitting of an ankle-foot orthosis in late stage stroke. (Hospital-based)

Each work package will evaluate the effectiveness of the visualisation software intervention on patient outcomes in a Phase II randomised clinical trial (exploratory) as defined by the MRC and will follow MRC guidelines for the evaluation of complex interventions [17].

To complement the quantitative outcome measures from the trials, qualitative data will be captured before, during and after the trial. This is essential for a complex intervention, in order to understand the determining factors in the quantitative outcome measures from the trial [18]. The qualitative research will explore the effects of the use of the tool on the experiences and understanding of both the patients and therapists separately, as well as the effect on the interactions between the different participants.

\section{Designing Biomechanical Visualisations: Addressing the Requirements of Different Rehabiliation Scenarios}

A different visualisation software tool is being developed for each work package to address the five specific clinical rehabilitation scenarios. In order to design the 
software to be appropriate to each scenario, there are a number of interrelated factors which need to be considered. These factors include:

- the rehabilitation setting

- the motion capture technologies employed

- the specific biomechanical parameters that are important (a) for clinical decision-making (b) to enable patients to better understand their tasks, goals and progress

- the patient's needs and experience of the rehabilitation process

The following sections illustrate some of the implications of these different factors for the design of the visualisations. The factors are discussed in relation to the three rehabilitation settings being investigated in the project - hospital, community and home.

\subsection{Biomechanical Visualisation in Hospital Rehabilitation}

Technology. In the hospital setting, the conditions to be assessed will be complex, requiring specialist treatment. There will be a fixed, laboratory setup providing fullbody optical motion capture and force plate measurements allowing for full kinematic, kinetic and spatiotemporal parameters to be collected. This system requires a high level of training to set up and operate. The system is expensive and not portable, however it does offer a high level of accuracy. An extensive range of biomechanical data are available for the visualisation software in this setting.

Biomechanical Parameters. The parameters of interest at this stage of the patient's rehabilitation will be those that are relevant to re-establishing the patient's functional capability (in contrast to the quality of the movement in the other scenarios).

There will be two different types of visualisation in this scenario: diagnostic, for the therapist; and explanatory, for the patient. The tool will need to be flexible in allowing the therapist to choose what visualisations are appropriate for different patients. Visualisations will be viewed off-line, as in this rehabilitation scenario it is important that the patient walks naturally and without any visual distractions.

For analysis, the visualisation tool needs to assist the therapist to interpret the complex and interrelated data which describe the movement. For example, two key progress measures for lower limb stroke rehabilitation are walking speed and symmetry of gait. The tool will enable the therapist to interactively select and view different biomechanical parameters relating to the outcome measures, to make more informed clinical decisions about the patient's rehabilitation. For communication to the patient, the visualisations will be able to be configured to enable the therapist to explain the various parameters measured, what they mean and how they relate to the patient's progress. In Fig. 1., the effect of step length on walking speed and gait symmetry are displayed in two different ways - the first for communication to the patient, the second for detailed analysis by the therapist. 


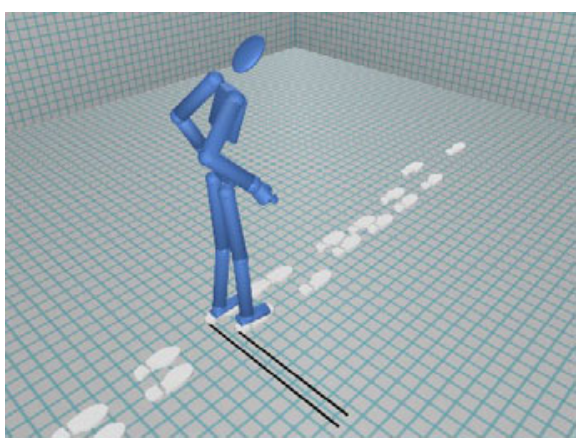

(a)

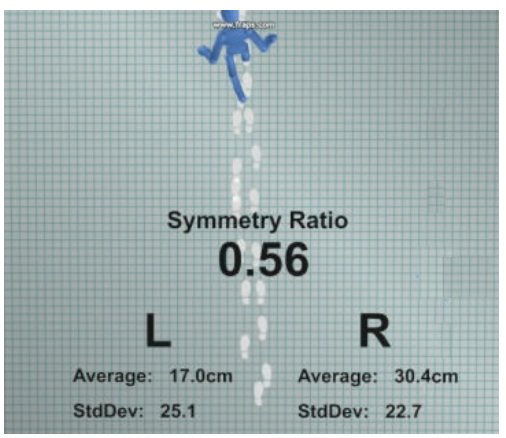

(b)

Fig. 1. (a) Example visualisation which visually shows the relationship between step length, gait symmetry and walking speed (b) Example visualisation which displays the same captured motion data, but provides diagnostic numerical data for the therapist

Patient Experience. Patients attending the rehabilitation sessions will be in the acute phase of their rehabilitation pathway, and will be more reliant on their therapist to lead the rehabilitation process. The patients will also find themselves in an unfamiliar setting.

In the design of the visualisation, sensitivity will be required in the representation of the figure and managing how the patient sees their own movements. For example, in the first session the patient may not have seen the severity of their movement problems in a clear objective way before. In fig. 2, the same movement is shown using different figure representations, each with different potential for an emotional response. The software will enable the representation of the figure to be chosen by the patient.

The patient's progress through their rehabilitation will also require to be sensitively handled. Progress through rehabilitation can be complex. There may be periods of rapid improvement, but also times where improvement is small, or there may be intermediate reductions in capability. The approach taken in the research to this sensitive area will be to make the tool flexible in how progress is communicated to the patient and give the therapist control over when and how progress is communicated. A potential advantage of the visualisation tool in this scenario is that the progress can be communicated by an objective tool, rather than by what may be conceived by the patient as the opinion of the therapist. The benefits this may provide for the interaction between the therapist and the patient will be explored in the research.

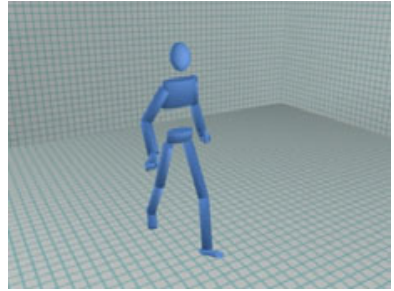

(a)

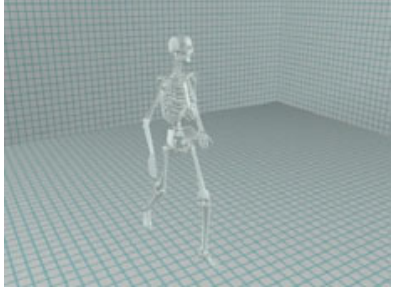

(b)

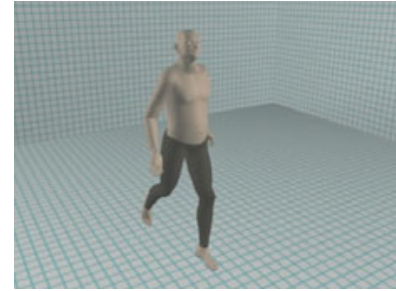

(c)

Fig. 2. (a) Stick Figure representation (b) Skeletal/Anatomical representation (c) Realistic representation 


\subsection{Biomechanical Visualisation in Community Rehabilitation}

Technology. In the community setting, the motion capture technology needs to be portable, and easy to setup and dismantle as they will mostly be used in shared spaces. The system will be setup and operated by the therapist, so this needs to be intuitive and quick to achieve. In order for the system to be feasible, it should be reasonably inexpensive. Low cost optical motion capture will be used for the lower limb stroke rehabilitation, and a wired electromagnetic system will be used for upper limb rehabilitation. Partial body motion capture will be used in this setting to enable quick setup of the equipment - one will capture only upper limb motion, the other only lower limb. This has obvious implications for what can be shown in the visualisation, as can be seen in Fig. 3 .

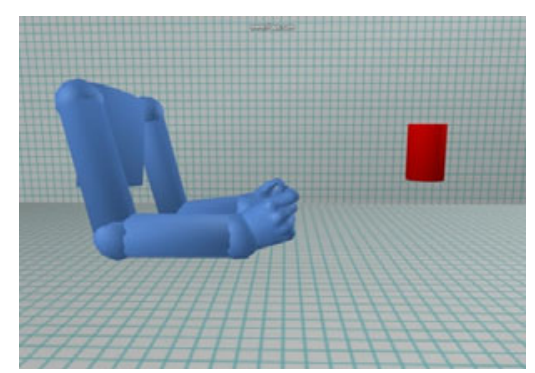

(a)

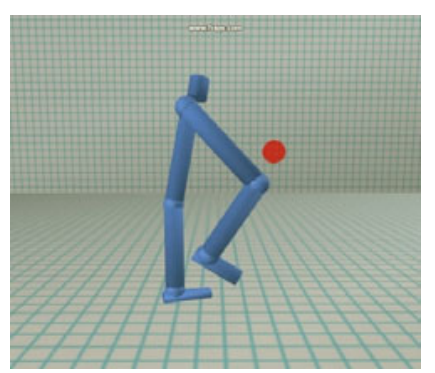

(b)

Fig. 3. Visualisations of partial-body motion capture. In each the participant is trying to reach for a virtual target (a) Upper limb and torso data only (b) Lower limb data only.

Biomechanical Parameters. The data collected and analysed by the therapist will be limited to kinematic and spatiotemporal parameters, and will be interpreted with a focus on quality of movement. There will be a greater emphasis on showing real-time data visualisations so the patient can receive instant feedback of their movements as they perform them. As in the hospital setting, the therapist will be on-hand to explain the visualisations to the patient and use them as a basis for discussion.

The rehabilitation tasks in the community setting are such that there are not necessarily distinct biomechanical goals defined in the literature with respect to the parameters measured. The goals have been determined by researchers engaging practicing therapists in the design process and unearthing the essence of each task.

Patient Experience. The patient will have been considered eligible to go home as part of early supported discharge. Their rehabilitation sessions will still be at regular intervals, and face-to-face with their therapist. The sessions will focus on increasing the quality of the movements rather than achieving functional goals.

The visualisations will focus on providing objective communication of compensatory movements which they may not be aware they are making. The reasons that the compensatory movements are reducing the effectiveness of the task will be visually demonstrated. 


\subsection{Biomechanical Visualisation in Home Rehabilitation}

Technology. The home is not a controlled environment, in contrast to a laboratory setting e.g. the size and layout of the room will vary; there will be different lighting conditions; other people may be present, not just the patient. This implies that the technology used needs to be simple, inexpensive and be able to set up without technical assistance. For this project, we will be using custom-made wearable wireless motion sensors. Feasibly the patients can only be asked to put on a small number of sensors, so that it is not onerous to use. The data on only selected segments of the body will be able to be measured and partial figures visualised. The patient's exercises can be recorded every session, providing many more measurements than can be achieved in e.g. a 1 hour session in a clinical setting.

Biomechanical parameters. The biomechanical parameters for home rehabilitation are very similar to those in the community setting - limited to kinematic and spatiotemporal and focused on quality of movement. They will additionally involve counting the number of repetitions, although the main difference is that there will be no therapist on-hand to explain the results. This means that the software design will need to place a greater emphasis on the pre-prepared explanatory material which explains clearly to the patient the biomechanical goals and objectives of each exercise.

Patient Experience. For home rehabilitation, the patient will be medically stable, and expected to self manage their use of the rehabilitation tool. The use of the tool will be voluntary in contrast to the other settings where the therapist will guide and encourage the patient. As the patient can use the tool to get immediate feedback at any time on their exercise performance and progress, how this is communicated will need to be handled carefully to avoid adversely affecting motivation and adherence.

The interaction with the therapist in the home will be less than in the hospital or the community (for the conditions investigated in this project, the level of contact with therapists is usually low). To address the reduction in patient-therapist interaction there are a number of options. Firstly, one could encapsulate some of the therapists' knowledge within the tool e.g. by creating rule-sets to automatically adjust goals for changes in performance and fatigue. Alternatively, one could establish a facility for regular remote communication with the therapist either live (via webcam/videoconferencing or phone), or offline using email. In conjunction with this, the recorded sensor data could be sent to the therapist to view using the same software tool so that both are looking at the same image.

\section{Conclusions}

The ability to apply and communicate biomechanical analysis to a wider range of rehabilitation settings than presently possibly could offer many benefits for both therapists and patients.

In order to achieve this, the research will provide visualisation software tools which support the analysis and interpretation of biomechanical data relevant to specific rehabilitation scenarios. The technical and practical limitations for the different 
rehabilitation settings will be explored in the research, to test the feasibility of the implementation of this approach.

The quantitative outcomes from the randomised controlled trials will test the hypothesis that use of visualisation software to communicate biomechanical concepts and measurements will result in better patient outcomes. Equally important to the study will be the qualitative research before, during and after the trials to explain the quantitative outcome measures i.e. which aspects of the use of the visualisations were successful or otherwise; whether the therapists better understood the biomechanical concepts and measurements, and whether this changed their diagnosis; the effect of the tool on the patient's understanding of their rehabilitation tasks, goals and progress; and any effect on the ability for the patients and therapists to communicate more clearly about the rehabilitation tasks and goals.

Acknowledgements. The authors would like to thank the greater envisage project team. This research is funded by the MRC Lifelong Health and Wellbeing programme (LLHW), grant number G0900583. LLHW is a cross council initiative in partnership with the UK health departments and led by the MRC.

\section{References}

1. Toro, B., Nester, C.J., Farren, P.C.: The status of gait assessment among physiotherapists in the United Kingdom. Arch. Phys. Med. Rehabil. 84, 1878-1884 (2003)

2. Baker, R.: Gait analysis methods in rehabilitation. J. NeuroEngineering Rehabil. 3, 1-10 (2006)

3. Coutts, F.: Gait analysis in the therapeutic environment. Manual Therapy 4, 2-10 (1999)

4. Simon, S.R.: Quantification of human motion: gait analysis - benefits and limitations to its application to clinical problems. Journal of Biomechanics 37, 1869-1880 (2004)

5. Kay, R.M., Dennis, S., Rethlefsen, S., Reynolds, R.A.K., Skaggs, D.L., Tolo, V.T.: The effect of preoperative gait analysis on orthopaedic decision making. Clin. Orthop. Rel. Res., 217-222 (2000)

6. Lofterod, B., Terjesen, T., Skaaret, I., Huse, A.B., Jahnsen, R.: Preoperative gait analysis has a substantial effect on orthopedic decision making in children with cerebral palsy Comparison between clinical evaluation and gait analysis in 60 patients. Acta Orthop. 78, 74-80 (2007)

7. Laine, C., Davidoff, F.: The patient physician relationship - Patient-centered medicine - A professional evolution. JAMA-J. Am. Med. Assoc. 275, 152-156 (1996)

8. Rosewilliam, S., Roskell, C.A., Pandyan, A.D.: A systematic review and synthesis of the quantitative and qualitative evidence behind patient-centred goal setting in stroke rehabilitation. Clin. Rehabil. 25, 501-514 (2011)

9. Wartman, S.A., Morlock, L.L., Malitz, F.E., Palm, E.A.: Patient Understanding and Satisfaction as Predictors of Compliance. Med. Care 21, 886-891 (1983)

10. Teutsch, C.: Patient-doctor communication. Med. Clin. N. Am. 87, 1115-1145 (2003)

11. Maclean, N., Pound, P., Wolfe, C., Rudd, A.: Qualitative analysis of stroke patients' motivation for rehabilitation. BMJ 321, 1051-1054 (2000)

12. Card, S., Mackinlay, J., Shneiderman, B.: Readings in Information Visualization: Using Vision to Think. Morgan Kaufmann Publishers, San Francisco (1999)

13. Wurman, R.S.: Information Anxiety 2. Que Publishing, Indianapolis (2000) 
14. Tufte, E.R.: The visual display of quantitative information. Graphics Press, Cheshire (1983)

15. Craig, P., Dieppe, P., Macintyre, S., Michie, S.: Developing and evaluating complex interventions: the new Medical Research Council guidance. BMJ 337 (2008)

16. Lewin, S., Glenton, C., Oxman, A.D.: Use of qualitative methods alongside randomised controlled trials of complex healthcare interventions: methodological study. BMJ 339 (2009)

17. Macdonald, A.S., Loudon, D., Rowe, P.J., Samuel, D., Hood, V., Nicol, A.C.: Towards a design tool for visualizing the functional demand placed on older adults by everyday living tasks. Universal Access in the Information Society 6, 137-144 (2007)

18. Macdonald, A.S., Loudon, D., Docherty, C., Miller, E.: Project findings: Innovation in envisioning dynamic biomechanical data to inform healthcare and design guidelines and strategy. New Dynamics of Ageing Programme, Sheffield (2009) 